\title{
The transition from 3C SiC(111) to graphene captured by Ultra High Vacuum Scanning Tunneling Microscopy
}

\author{
B.Gupta1 ${ }^{1}$ E.Placidi' ${ }^{2}$ C.Hogan' ${ }^{2}$, N.Mishra ${ }^{3}$, F.Iacopi ${ }^{3}$, N.Motta*1 \\ ${ }^{1}$ School of Chemistry Physics and Mechanical Engineering and Institute for Future \\ Environments, Queensland University of Technology, 2 George Street, Brisbane 4001, QLD, \\ Australia \\ ${ }^{2}$ Istituto di Struttura della Materia CNR, Via del Fosso del Cavaliere 00133 Roma and \\ Università di Roma "Tor Vergata", dipartimento di Fisica, via della Ricerca Scientifica 1, \\ 00133 Roma. \\ ${ }^{3}$ Queensland Micro and Nanotechnology Centre, Griffith University, Nathan Campus 4111, \\ QLD Australia
}

\begin{abstract}
In this paper we clarify the transformation mechanism of 3C-SiC into graphene upon thermal decomposition, by a combination of high resolution Scanning Tunneling Microscopy (STM) images and first principle calculations. We studied the transition from 3C-SiC to graphene by high temperature annealing of C-terminated 3C SiC(111)/Si(111) samples in Ultra High Vacuum. By using STM we were able to observe very clear atomic resolution images of the transition from $\operatorname{SiC}(\sqrt{3} \times \sqrt{3}) R 30^{\circ}$ to a new intermediate stage $\operatorname{SiC}\left(\frac{3}{2} \times \sqrt{3}\right) R 30^{\circ}$ (very close to the graphene $(2 \times 2)$ reconstruction) after annealing at $1250^{\circ} \mathrm{C}$. We also obtained images of the transformation of the intermediate structure into a $(1 \times 1)$ monolayer graphene, caused by further sublimation of atoms in the subsurface layer. We have interpreted the results by using Density Functional Theory - Local Density Approximation calculations, which give full account of the $\mathrm{SiC}(\sqrt{3} \times \sqrt{3}) R 30^{\circ}$ reconstruction, but fail to describe the $\mathrm{SiC}\left(\frac{3}{2} \times \sqrt{3}\right) R 30^{\circ}$ structure due to its incommensurability with the 3C-SiC(111) lattice.
\end{abstract}

*Corresponding Author. Email: n.motta@qut.edu.au.(N.Motta) Tel +61 731385104 


\section{Introduction}

The growth of graphene by selective Si sublimation on Silicon Carbide (SiC) substrates is well documented [1-7]. This technique provides a reliable way to obtain a controlled and continuous epitaxial graphene layer $[8,9]$ leading to wafer size material for large scale device production. Even more important is the growth of graphene on $\mathrm{SiC} / \mathrm{Si}$ substrates, to combine the low cost of $\mathrm{Si}$ with the epitaxial growth of graphene on $\mathrm{SiC}$ [10-13]. The considerable advantage of this method is the perspective of achieving perfect integration of graphene with the electronic circuitry developed on Si by using the traditional microelectronic platform. In order to achieve a full control of this technique it is of utmost importance to fully understand the steps leading to the formation of graphene from SiC. While the different reconstructions which appear on the $\mathrm{SiC}$ surface are well known, the details of the transformation from $\mathrm{SiC}$ to graphene are not completely clear, in spite of the large number of Scanning Tunneling Microscopy (STM) studies reporting atomic resolution images [1-7, 14-17].

The analysis of the graphitic structures obtained by high temperature annealing of SiC and its different stages of reconstruction have been studied by Low Energy Electron Diffraction (LEED) since 1975 [18]. It has been recognized that the reconstructions leading to graphene are different for silicon (Si)-terminated and carbon (C)-terminated faces of $6 \mathrm{H}$ and $4 \mathrm{H} \mathrm{SiC}$, but it can be applied also to 3C SiC which has the same stacking in the top layers.

For the Si-terminated face the reconstruction proceeds by increasing the annealing temperature through $(3 \times 3),(\sqrt{3} \times \sqrt{3}) R 30^{\circ},(6 \sqrt{3} \times 6 \sqrt{3}) R 30^{\circ}$ and graphene $(1 \times 1)$. $(3 \times 3)$ is a Si-rich phase and a very stable structure which consists of a complete $\mathrm{Si}$ 
adlayer on top of an uppermost bulk like SiC substrate layer which is usually obtained by annealing at $850^{\circ} \mathrm{C}$ under Si flux. The adlayer contains no vacancies or corner holes, and it is covered by tetrahedral adatom clusters with three Si base atoms and one top Si atom per unit cell [19]; the base Si trimer lies on a twisted Si adlayer forming cloverlike rings on the Si-terminated face. This $(3 \times 3)$ translational symmetry is present under very Si-rich conditions [19], while the alternative $(\sqrt{3} \times \sqrt{3}) R 30^{\circ}$ reconstruction is favoured by less Si-rich preparation conditions[16]. The amount of Si supply and the heating time and temperature thus determine the reconstruction of SiC before the graphene formation [20-22]. The $(6 \sqrt{3} \times 6 \sqrt{3}) R 30^{\circ}$ phase has a complicated surface reconstruction and consists of $(13 \times 13)$ unit cells of graphene. This surface reconstruction is attributed to a C-rich phase but does not have any graphitic properties, as the adlayer has a strong interaction with the substrate, and so it is considered a buffer layer or an interface layer. The buffer layer passivates the SiC surface so that the subsequent $\mathrm{C}$ planes are only weakly bound to the substrate [23-25]. Finally, the $(1 \times 1)$ graphene surface structure appears when the formation of graphene layer is complete on the surface. The above surface reconstructions do not depend on the SiC polytype, and they have been been found on all the hexagonally arranged surfaces of 3C, $4 \mathrm{H}$ and $6 \mathrm{H} \mathrm{SiC}[23,26-31]$.

On the C-terminated face the $(6 \sqrt{3} \times 6 \sqrt{3}) \mathrm{R} 30^{\circ}$ reconstruction is not observed, while the $(\sqrt{3} \times \sqrt{3}) R 30^{\circ}$ has been rarely reported [5]. The most accepted sequence of graphene growth on the C-terminated face is $(2 \times 2)_{S i},(3 \times 3),(2 \times 2)_{C}$ and $(1 \times 1)$ graphene $[6,23$, $24,32-34]$. The two phases $(2 \times 2)_{S i}$ and $(2 \times 2)_{C}$ occur from different surface treatments leading to $\mathrm{Si}$ and $\mathrm{C}$ rich structures respectively $[23,34]$. The $(2 \times 2)_{S i}$ phase develops upon annealing in Si flux. This procedure also helps to remove surface oxides from the 
SiC samples $[13,23]$. Further annealing of the surface leads towards $(3 \times 3)$ and $(2 \times 2)_{C}$ surface reconstructions $[6,23]$ and finally to graphene growth.

In the case of the $\mathrm{Si}$ face of $\mathrm{SiC}(0001)$, the graphitization process is slow, and it is easy to control the number of graphene layers, while for a C-terminated (000-1) surface the graphitization process is very fast and large number of graphene layers are formed (up to 100). The resulting electron mobility, however turns out to be low for the Si face and high for the C face [35].

While excellent graphene quality has been achieved in the last few years by annealing bulk SiC $[8,9]$, the cost of the substrate compared to Si and the limited diameter of the wafers are a stumbling block towards large industrial-scale fabrication of graphene from bulk SiC. To overcome these problems, 3C SiC epitaxially grown on Si wafers has been proposed as a suitable substrate for the synthesis of epitaxial graphene (EG) [10, 12, 36-39]. The most suitable surface in this case is 3C SiC (111) as its top four layers are identical to those of $6 \mathrm{H} \mathrm{SiC}(0001)$ (Fig-1).

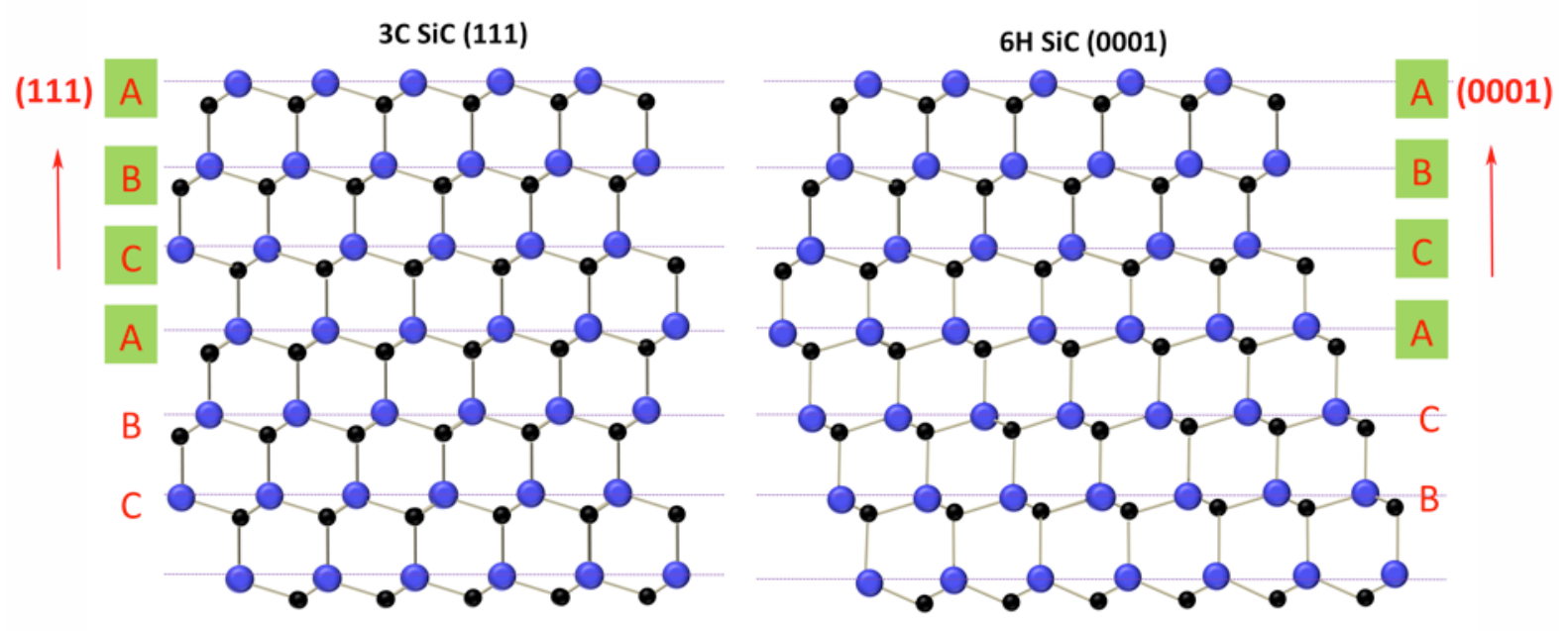

Figure 1: Cross section of the 3C SiC(111) structure (left) and 6H SiC(0001) (right) showing the different layer stacking 
3C SiC (111)/Si (111) has also two polar faces (as $6 \mathrm{H}$ and $4 \mathrm{H}$ ). Little attention has been given to the $\mathrm{C}$-face while the $\mathrm{Si}$-face has been studied comparably well. Graphene on $\mathrm{Si}$ terminated 3C $\mathrm{SiC} / \mathrm{Si}$ (111) follows the same sequence of reconstuctions towards graphene as on $6 \mathrm{H} \mathrm{SiC}(0001)$ or $4 \mathrm{H} \mathrm{SiC}(0001)[6,28,39,40]$. As mentioned above, the well known reconstruction phases on these polytypes are $(3 \times 3),(\sqrt{3} \times \sqrt{3}) R 30^{\circ}$, $(6 \sqrt{3} \times 6 \sqrt{3}) R 30^{\circ}$ and graphene $(1 \times 1)$ [22, 23, 40-43]. Recently Darakchieva et al [44] reported on the reconstruction of the $\mathrm{C}$ face of $3 \mathrm{C} \mathrm{SiC}$ (111), confirming by LEED the absence of the $(6 \sqrt{3} \times 6 \sqrt{3}) \mathrm{R} 30^{\circ}$ phase as in $6 \mathrm{H}$ and $4 \mathrm{H} \mathrm{SiC}$.

Thermal decomposition of heteroepitaxially grown 3C SiC/ Si (111) for EG is possible in Ultra High Vacuum (UHV) [8] at temperatures lower than $1400^{\circ} \mathrm{C}$, below the melting point of Si. UHV growth results also in a contaminant free surface, providing a perfect starting material for nanoelectronic applications and devices.

In spite of the number of studies reported for EG on $3 C \operatorname{SiC}[10-13,28,38,45,46]$, so far the details of the atomic transformation leading to the formation of graphene have not been clarified.

In the present work we analyse by STM the transformation of 3C-SiC(111) in graphene caused by high temperature annealing in UHV. We have been able to capture high resolution images of the different kind of reconstructions leading to graphene formation. We explain the sequence for the first time with the help of Density Functional Theory - Local Density Approximation (DFT-LDA) calculations, thus achieving a full picture of the reconstructions and graphene growth on 3C SiC (111). 


\section{Experiment}

A $250 \mathrm{~nm}$ thick layer of 3C SiC (111) was grown on Si (111) by the alternating supply epitaxy method [47]. Our 3C-SiC/Si(111) samples are preferentially C-terminated by the CVD growth process[47], although due to stacking faults and defects this termination is not guaranteed. As received samples were cut to the size of $(1.5 \times$ 12) $\mathrm{mm}^{2}$ for STM measurement, cleaned by 5 mins of ultrasonication with isopropanol, 2 mins by deionised water and then dried by $\mathrm{N}_{2}$ gas. The samples were then introduced into our UHV Omicron system (base pressure in $10^{-11}$ mbar range) equipped with a Variable Temperature XA STM . After degassing for a few hours at $600^{\circ} \mathrm{C}$, the samples were annealed $10^{\prime}$ by direct current heating at $1250^{\circ} \mathrm{C}$ for the graphene formation, leading to an average of 3 graphene layers as determined by XPS [13, 48]. However, due to the roughness of the $\mathrm{SiC}$ surface we expect to find regions where the number of layers is larger or smaller. The temperature was measured by a pyrometer (IRCON Ultimax Plus UX 20P) with an uncertainty of about $10^{\circ} \mathrm{C}$. The sample was left in UHV for 30 mins to cool down, and then transferred to the STM stage. Tungsten tips used for STM characterization were fabricated by electrochemical etching.

\section{Results}

Based on our STM images we found that the transition occurs in two subsequent steps:

1. Transition of SiC surface from $(\sqrt{3} \times \sqrt{3}) R 30^{\circ}$ to $\left(\frac{3}{2} \times \sqrt{3}\right) R 30^{\circ} .{ }^{1}$

2. Transition from $\left(\frac{3}{2} \times \sqrt{3}\right) R 30^{\circ}$ to monolayer graphene.

\footnotetext{
${ }^{1}$ We will show later that $\left(\frac{3}{2} \times \sqrt{3}\right) R 30^{\circ}$ reconstruction fit more with a quasi $(2 \times 2)$ symmetry of graphene, but we will maintain this notation for a straightforward comparison with the close $(\sqrt{3} \times \sqrt{3}) R 30^{\circ}$ symmetry commensurate with $\operatorname{SiC}(111)$ surface.
} 


\subsection{Transition from $(\sqrt{3} \times \sqrt{3}) \mathbf{R}^{\circ} 0^{\circ}$ to $\left(\frac{3}{2} \times \sqrt{3}\right) \mathbf{R 3 0}^{\circ}$}

In Figure 2(a) we observe the coexistence of two different reconstructions, with a progressive transition from one to the other. We follow the transiton going from the right to the left in the image, as we believe this is the direction of the transformation. To enhance the effect we have drawn circles around the brighter atoms in figure 2(b), with black circles in the left region and blue circles in the right region. At the right side of the image we notice the formation of hollow hexagons with 3 brighter and 3 darker atoms. The holes at the centre of the hexagons and the brighter atoms form a quite regular hexagonal centered network with a lattice constant of 5.3 A (Fig 2c), very close to $(\sqrt{3} \times a)=5.35 \AA$, indicating a $(\sqrt{3} \times \sqrt{3})$ R30 surface recostruction of the SiC.

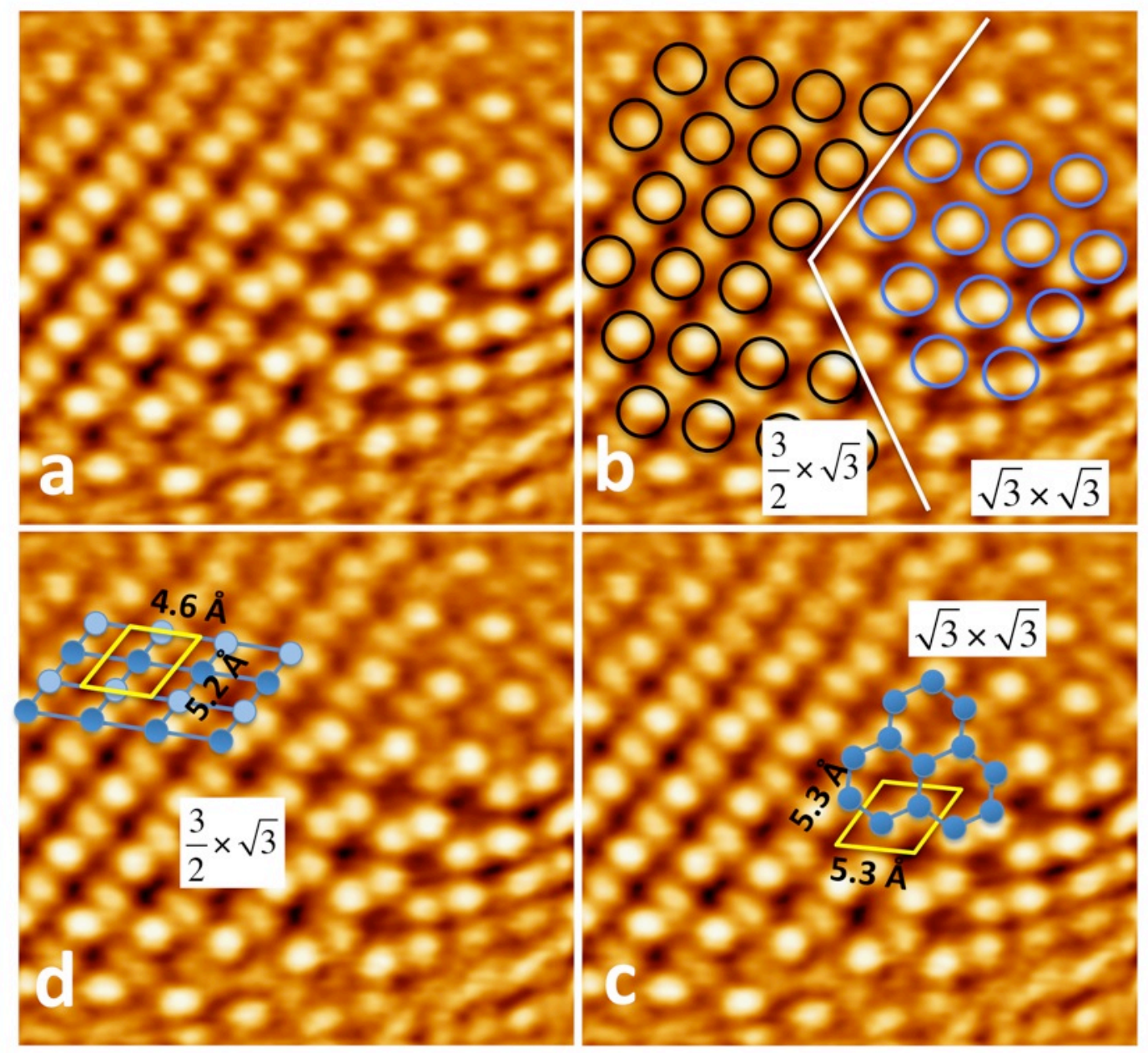


Figure 2: STM images of $3 \mathrm{C} \mathrm{SiC}(111) / \mathrm{Si}(111)$ after annealing at $1250{ }^{\circ} \mathrm{C}$. (a) $(3.6 \times 3.6) \mathrm{nm}^{2}$ STM image $(\mathrm{V}=60 \mathrm{mV} \mathrm{I}=80 \mathrm{pA})$ showing the coexistence of two different reconstructed phases. (b) Same image with black and blue circles around the brighter atoms to indicate the two different reconstructions. (c) the $(\sqrt{3} \times \sqrt{3}) \mathrm{R} 30^{\circ}$ reconstruction $(\mathrm{d})$ the $\left(\frac{3}{2} \times \sqrt{3}\right) \mathrm{R} 30^{\circ}$ reconstruction. The panels are labeled following the direction of reconstruction.

On the left side of the image the central holes and the hexagons have shrunk and the atoms now appear arranged in parallel lines with alternated dark and bright atoms. The periodicity along these lines is $5.2 \AA$. The two bright atoms across the lines are spaced by $4.6 \AA$, matching a nominal $\left(\frac{3}{2} \times \sqrt{3}\right) \mathrm{R} 30^{\circ}$ surface reconstruction (fig $2 \mathrm{~d}$ ). The smoothness of the transition from one reconstruction to the other is clearly shown in Fig.3, where the profile of two nearby lines of atoms indicates the shrinking of the interatomic distance going from right to left. In fact the periodicity along line 2 changes from 5.3 to $4.6 \AA$ (coherently reading from right to left) while along line 1 the length of the oscillations is constant, equal to $4.6 \AA$. In fact, looking at the orientation of the twoatoms element identifying the cell, one can notice their axis progressively twisting by $30^{\circ}$.
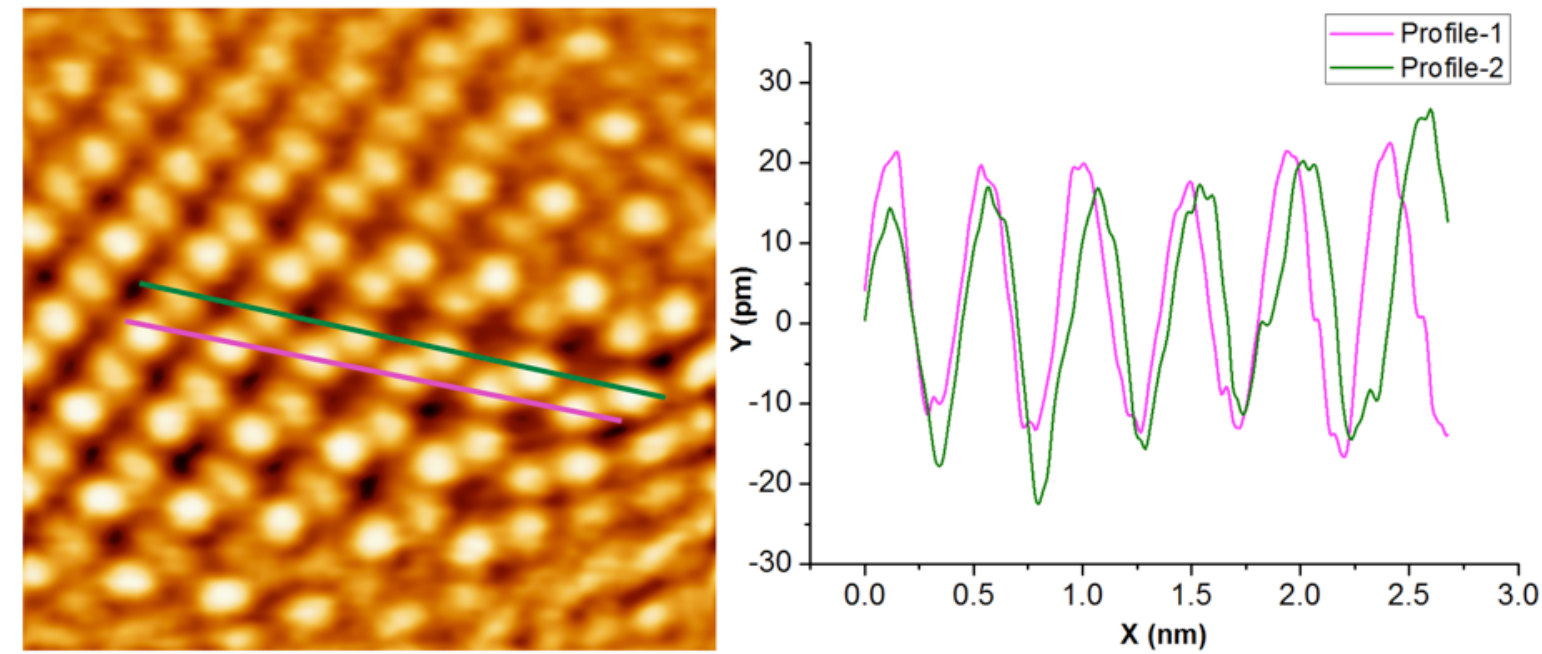

Figure 3: Profiles of the STM image in Fig 2, showing the change in the atomic positions: going from right to left the distance of profile-2 (green) is gradually decreasing until matching that of profile-1 (purple), which remains nearly same. 
We have simulated the $(\sqrt{3} \times \sqrt{3})$ reconstruction of $\mathrm{SiC}$ by using density functional theory in the local density approximation (DFT-LDA) and a plane wave/pseudo potential scheme [49]. Surfaces were modelled using slabs containing $6 \mathrm{SiC}$ (111) bilayers, back terminated with hydrogen, allowing the front most 4 atomic layers to relax. Hydrogen is used for the saturation of the back terminated part in order to get rid of dangling bonds which could give rise to spurious interactions or charge transfer between the two slab surfaces. STM images were computed at constant current using the Tersoff - Hamann approximation [50].
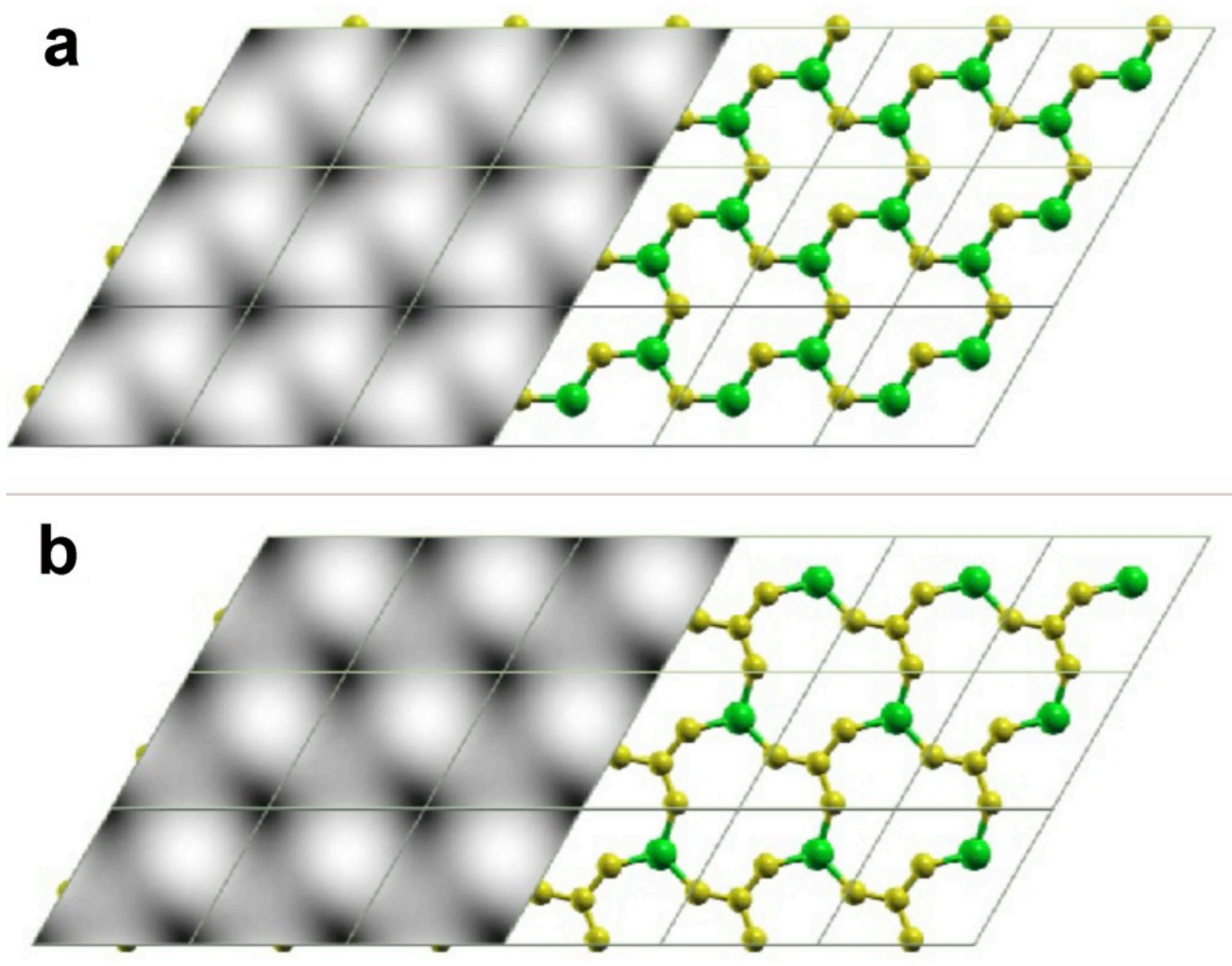

Figure 4: Simulated STM images of the $(\sqrt{3} \times \sqrt{3})$ phase using two different configurations: a) one missing Si atom per unit cell; b) with a $\mathrm{C}$ atom substituting a Si atom. Si atoms: larger in green; $\mathrm{C}$ atoms: smaller, in yellow 
Figure 4 shows computed STM images of the bulk truncated 3C SiC(111) surface alongside models of the relaxed geometries (only the top layer is shown) featuring one missing Si atom per $(\sqrt{3} \times \sqrt{3})$ unit cell (a) and additionally with a $\mathrm{C}$ atom substituting a Si atom (b). The bottom image resembles very closely the observed image for the $(\sqrt{3} \times \sqrt{3})$ reconstruction (see Fig 2$)$ and thus reflects the onset of the transformation of $\mathrm{SiC}$ into graphene.

We were not able to obtain a stable structural model of the $\left(\frac{3}{2} \times \sqrt{3}\right)$ R30 $0^{\circ}$ reconstruction, due to the fact that this is incommensurate to the $3 \mathrm{C} \mathrm{SiC} \mathrm{(111)} \mathrm{surface} \mathrm{cell,} \mathrm{and} \mathrm{it} \mathrm{is}$ thereby difficult to simulate within periodic boundary conditions. We interpret the incommensuration with the $\mathrm{SiC}$ surface as an indication of the transition to graphene, speculating on a possible detachment of the topmost layer from the bulk. Such a layer might not be stable when connected to the bulk due to the awkward coordination with the 3C SiC (111) cell underneath, while the detachment may help to lower the elastic energy. For the sake of completeness we show a tentative model for the $(\sqrt{3} \times \sqrt{3}) \mathrm{R} 30^{\circ}$ phase, the $\left(\frac{3}{2} \times \sqrt{3}\right) \mathrm{R} 30^{\circ}$ phase and the transition between them (Fig 5). It is evident how the $(\sqrt{3} \times \sqrt{3}) \mathrm{R} 30^{\circ}$ closely matches the SiC structure, while the $\left(\frac{3}{2} \times \sqrt{3}\right) \mathrm{R} 30^{\circ}$ is out of registry ${ }^{2}$.

\footnotetext{
2 One could speculate that the atomic composition gradually changes through further sublimation, which eventually leads to instability and detactment of the layer, e.g. Fig4a is the root(3), and Fig4b is a sort of strained $3 / 2$ reconstruction before detachment of the upper layer.
} 


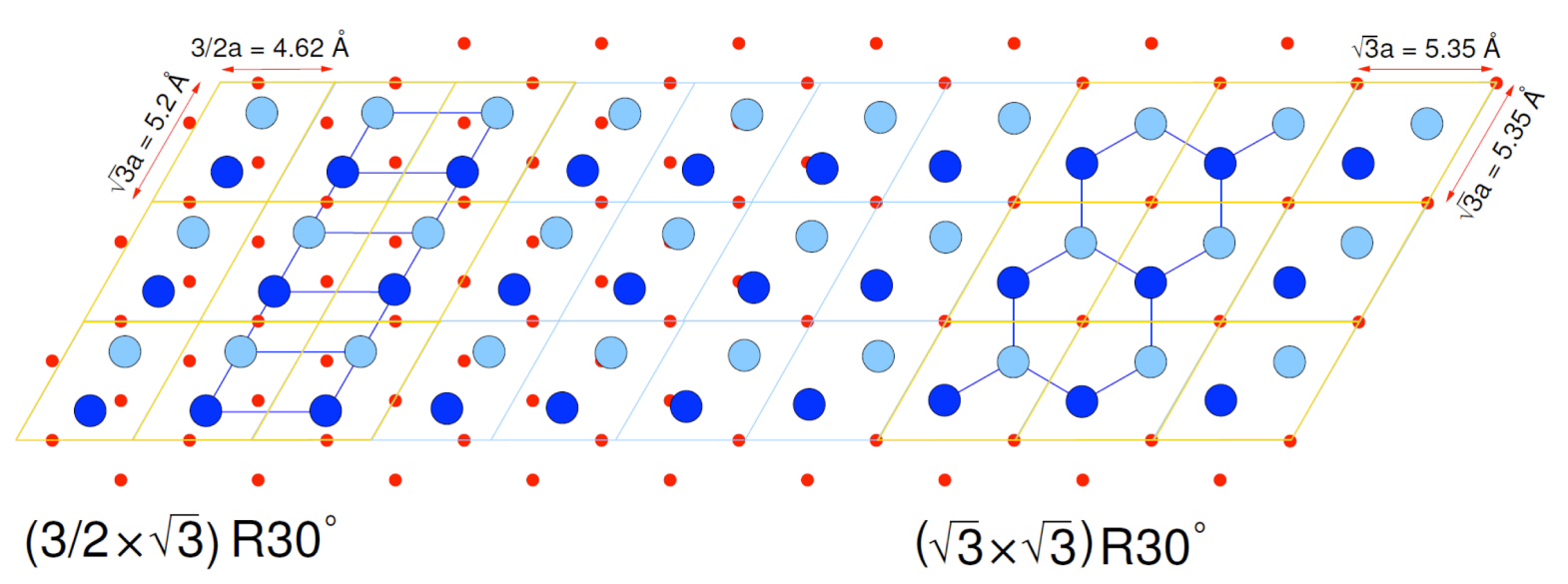

Figure 5: Transformation model for $(\sqrt{3} \times \sqrt{3})$ R $30^{\circ}$ towards $\left(\frac{3}{2} \times \sqrt{3}\right)$ R $30^{\circ}$ unit cell starting from right and going to towards left. The unit cells are represented in blue. Small red dots represent the $(1 \mathrm{x} 1)$ cell vertices of 3C SiC/Si (111), with a unit vector equal to $a$. Dots of light and dark blue represent the reconstructed atoms of higher and lower intensities on the $3 \mathrm{C}$ SiC. The rotation angle between the surface atoms and the bulk is $30^{\circ}$.

\subsection{Transition from $\left(\frac{3}{2} \times \sqrt{3}\right)$ to graphene}

In figure 6 (a) we notice a residual of the $\left(\frac{3}{2} \times \sqrt{3}\right)$ reconstruction evidenced by the left yellow rhombus in fig 6 (c). By measuring carefully the bright atoms distances we find that the cell parameter is now $4.9 \AA$ instead of $5.2 \AA$, This last distance corresponds to the graphene $2 \times 2$ reconstruction, and strengthens our hypothesis that the $\left(\frac{3}{2} \times \sqrt{3}\right)$ is a precursor of the graphene formation. Figure 7a shows a tentative overlap of a $(4.6 \times 5.2) \AA^{2}$ cell with the graphene $(2 \times 2)$ lattice cell. If we consider a $(2 \times 2)$ supercell of graphene, we obtain a $(4.9 \times 4.9) \AA^{2}$ rhombus. From the picture it appears clearly that the $\left(\frac{3}{2} \times \sqrt{3}\right) \mathrm{R} 30^{\circ}$ cell can be fitted to the graphene $(2 \times 2)$ with a little compressive distortion. The surface ratio between $\left(\frac{3}{2} \times \sqrt{3}\right) \mathrm{R} 30^{\circ}$ and a $(2 \times 2)$ graphene is around 0.99, i.e. an area compression of only $1 \%$. The ratio of $(\sqrt{3} \times \sqrt{3}) \mathrm{R} 30^{\circ}$ to the graphene lattice is instead 1.18 and it would require a high distortion energy, which makes the transition from $(\sqrt{3} \times \sqrt{3}) \mathrm{R} 30^{\circ}$ to graphene very expensive from an energetic point of view. 
Because of this relaxation we label the reconstruction as $(2 \times 2)$ in fig 6 . It must be noticed as well that, going from the bottom to the top of the image in fig 6a, some atoms gradually disappear from the reconstruction, both from the topmost layer and from the layer below. At the top right end it is possible to see clearly the $(1 \times 1)$ cell of the monolayer graphene (hexagonal structure), with a periodicity of $2.45 \AA$ (Fig 6b). The relaxation of the $\left(\frac{3}{2} \times \sqrt{3}\right)$ phase to a graphene $(2 \times 2)$ reconstruction is likely to be related to a further Si sublimation that disconnects locally the topmost layer from the bulk and allows the reconstruction to match the graphene symmetry.
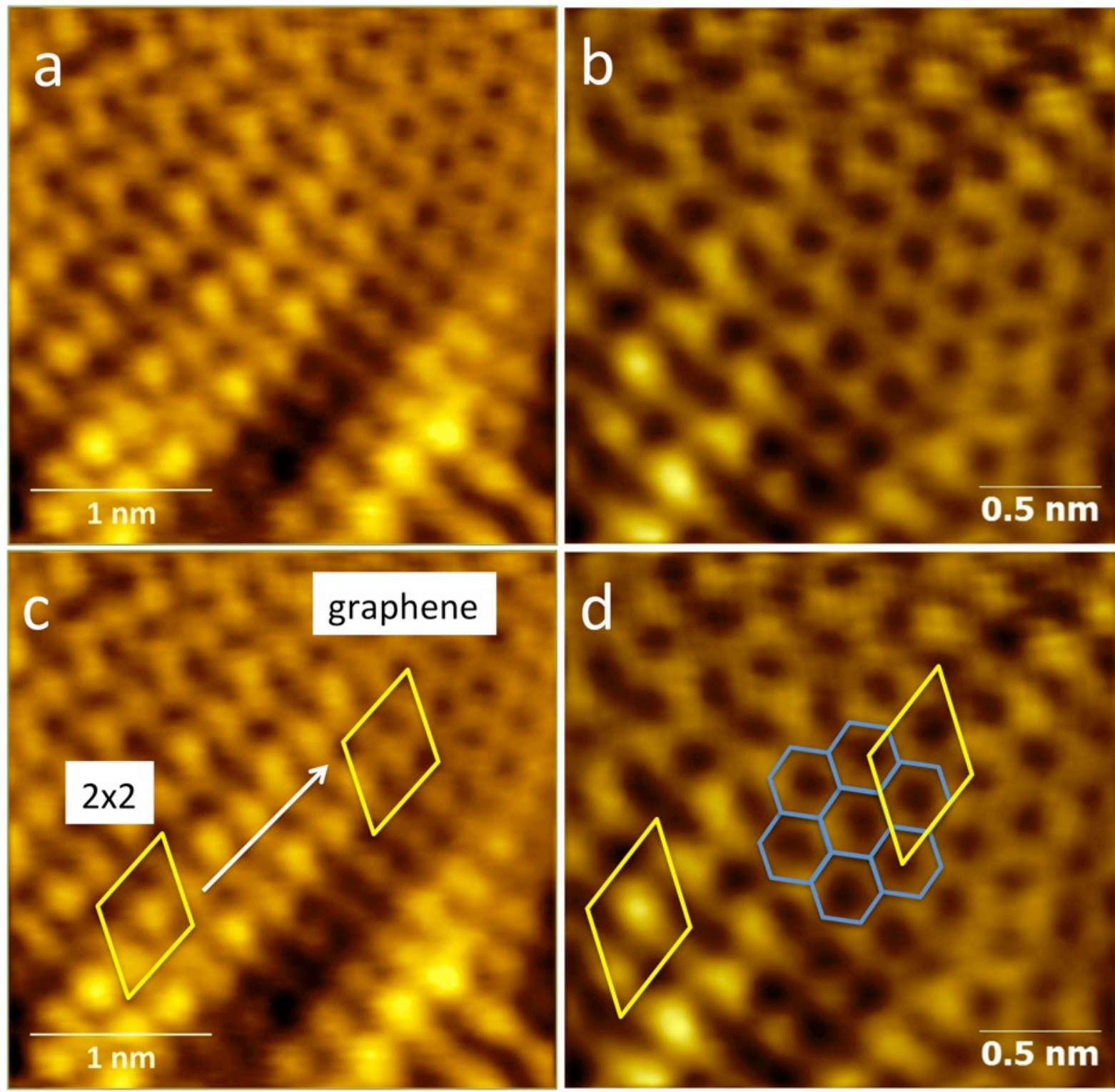
Figure 6: Transformation of the $\left(\frac{3}{2} \times \sqrt{3}\right) \mathrm{R} 30^{\circ}$ unit cell into a $(2 \times 2)$ cell and to graphene. (a) STM image (bias-0.02V; current-80pA) of the $(2 \times 2)$ region (former $\left(\frac{3}{2} \times \sqrt{3}\right) \mathrm{R} 30^{\circ}$ ) where it is possible to see the transformation going from bottom left to top right. (b) STM close up image of the graphene region (bias-0.02V; current-80pA) (c) Image (a) with the $(2 \times 2)$ structures superimposed. (d) Image (b) where the blue hexagons with a $2.45 \AA$ periodicity indicate the graphene area. The yellow rhombus in the same area compares the $(2 \times 2)$ structure with the graphene hexagonal network.

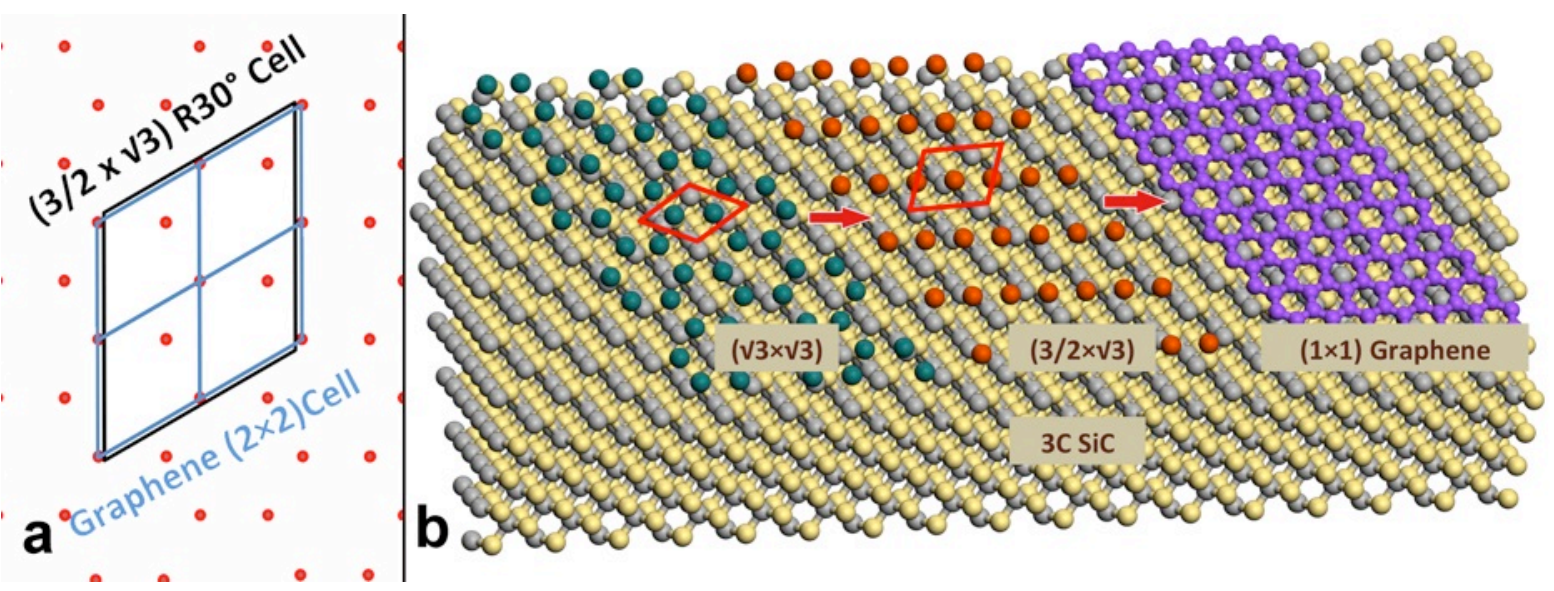

Figure 7: Model of the SiC to graphene transformation. a) A comparision between $\left(\frac{3}{2} \times \sqrt{3}\right) \mathrm{R} 30^{\circ}$ unit cell (black) and a graphene $(2 \times 2)$ cell (blue). b) a schematics of the transformation from $(\sqrt{3} \times \sqrt{3}) \mathrm{R} 30^{\circ}$ to $\left(\frac{3}{2} \times \sqrt{3}\right) \mathrm{R} 30^{\circ}$ into graphene $(1 \times 1)$

In summary, the $(\sqrt{3} \times \sqrt{3}) \mathrm{R} 30^{\circ}$ phase still matches the $3 \mathrm{C}$ SiC surface while the $\left(\frac{3}{2} \times\right.$ $\sqrt{3}) \mathrm{R}^{\circ} 0^{\circ}$ is actually a distorted $(2 \times 2)$ graphene, which is likely to be induced by the $\mathrm{Si}$ atoms, i.e. by their residual $\mathrm{sp}^{3}$ hybridization. Because of the Si atoms desorption the distances converge firstly to a $(2 \times 2)$ phase and finally to a $(1 \times 1)$ graphene layer, once the Si desorption has completed. A sketch of the full transition is depicted in fig $7 \mathrm{~b}$.

\section{Conclusion}

We performed a thorough STM study of the transformation of 3C-SiC(111) to graphene by high temperature annealing in UHV. By using a combination of STM and first principle calculations two main reconstructions have been recognized on SiC: a 
$(\sqrt{3} \times \sqrt{3}) \mathrm{R}^{\circ} 0^{\circ}$ and $\mathrm{a}\left(\frac{3}{2} \times \sqrt{3}\right) \mathrm{R} 30^{\circ}$. We suggest that these reconstructions are caused by the decrease of the density of Si atoms on the surface. The $\left(\frac{3}{2} \times \sqrt{3}\right) \mathrm{R} 30^{\circ}$ phase, which does not match well with the SiC cell, is actually a slightly distorted graphene $(2 \times$ 2 ), indicating the pathway for the transformation. The sublimation of more Si atoms leads in fact to the appearance of graphene $(1 \times 1)$ as confirmed by the STM images.

In conclusion we believe that our atomic resolution images of the sequence of reconstructions on 3C-SiC(111) provide for the first time a clear picture of the 3C$\mathrm{SiC}(111)$ to graphene transformation. However, as the stacking sequence of the first four atomic planes in $3 \mathrm{C} \mathrm{SiC(111)} \mathrm{is} \mathrm{similar} \mathrm{to} \mathrm{that} \mathrm{of} 6 \mathrm{H}$ and $4 \mathrm{H} \mathrm{SiC} \mathrm{(0001),} \mathrm{the} \mathrm{validity}$ of our findings can be extended also to the $6 \mathrm{H}$ and $4 \mathrm{H}$ phases.

\section{Acknowledgements:}

The author acknowledge the support of the Australian Research Council (ARC) through the Discovery project DP130102120, the Marie Curie International Research staff Exchange Scheme Fellowship within the $7^{\text {th }}$ European Community Framework Programme, and the Australian National Fabrication Facility. FI is recipient of a Future Fellowship from the Australian Research Council (FT120100445). The technical support of Dr. Peter Hines at the Central Analytical Research Facility of the Institute of Future Environments as well as the funding by the ARC through the LIEF grant (LE100100146) are also kindly acknowledged. CH acknowledges supercomputing resources at CINECA through the ISCRA initiative. 


\section{References}

[1] Berger, C., Z. Song, T. Li, X. Li, A.Y. Ogbazghi, R. Feng, Z. Dai, A.N. Marchenkov, E.H. Conrad, P.N. First, and W.A. de Heer, Ultrathin Epitaxial Graphite: 2D Electron Gas Properties and a Route toward Graphene-based Nanoelectronics. The Journal of Physical Chemistry B, 2004. 108(52): p. 19912-19916.

[2] de Heer, W.A., C. Berger, X. Wu, P.N. First, E.H. Conrad, X. Li, T. Li, M. Sprinkle, J. Hass, M.L. Sadowski, M. Potemski, and G.r. Martinez, Epitaxial graphene. Solid State Communications, 2007. 143(1-2): p. 92-100.

[3] Mallet, P., F. Varchon, C. Naud, L. Magaud, C. Berger, and J.Y. Veuillen, Electron states of mono- and bilayer graphene on SiC probed by scanning-tunneling microscopy. Physical Review B, 2007. 76(4): p. 041403.

[4] Riedl, C., U. Starke, J. Bernhardt, M. Franke, and K. Heinz, Structural properties of the graphene-SiC(0001) interface as a key for the preparation of homogeneous large-terrace graphene surfaces. Physical Review B, 2007. 76(24): p. 245406.

[5] Varchon, F., R. Feng, J. Hass, X. Li, B.N. Nguyen, C. Naud, P. Mallet, J.Y. Veuillen, C. Berger, E.H. Conrad, and L. Magaud, Electronic Structure of Epitaxial Graphene Layers on SiC: Effect of the Substrate. Physical Review Letters, 2007. 99(12): p. 126805.

[6] Emtsev, K., F. Speck, T. Seyller, L. Ley, and J. Riley, Interaction, growth, and ordering of epitaxial graphene on SiC $\{0001\}$ surfaces: A comparative photoelectron spectroscopy study. Physical Review B, 2008. 77(15): p. 155303.

[7] Huang, H., W. Chen, S. Chen, and A.T.S. Wee, Bottom-up Growth of Epitaxial Graphene on 6H-SiC(0001). Acs Nano, 2008. 2(12): p. 2513-2518.

[8] Emtsev, K.V., A. Bostwick, K. Horn, J. Jobst, G.L. Kellogg, L. Ley, J.L. McChesney, T. Ohta, S.A. Reshanov, and J. Röhrl, Towards wafer-size graphene layers by atmospheric pressure graphitization of silicon carbide. Nature materials, 2009. 8(3): p. 203-207.

[9] Ouerghi, A., M.G. Silly, M. Marangolo, C. Mathieu, M. Eddrief, M. Picher, F. Sirotti, S. El Moussaoui, and R. Belkhou, Large-Area and High-Quality Epitaxial Graphene on Off-Axis SiC Wafers. Acs Nano, 2012. 6(7): p. 6075-6082.

[10] Ouerghi, A., M. Marangolo, R. Belkhou, S. El Moussaoui, M.G. Silly, M. Eddrief, L. Largeau, M. Portail, B. Fain, and F. Sirotti, Epitaxial graphene on 3C-SiC(111) pseudosubstrate: Structural and electronic properties. Physical Review B, 2010. 82(12): p. 125445.

[11] Ouerghi, A., R. Belkhou, M. Marangolo, M. Silly, S. El Moussaoui, M. Eddrief, L. Largeau, M. Portail, and F. Sirotti, Structural coherency of epitaxial graphene on 3C-SiC (111) epilayers on Si (111). Applied Physics Letters, 2010. 97: p. 161905. 
[12] Ouerghi, A., M. Ridene, A. Balan, R. Belkhou, A. Barbier, N. Gogneau, M. Portail, A. Michon, S. Latil, P. Jegou, and A. Shukla, Sharp interface in epitaxial graphene layers on 3CSiC(100)/Si(100) wafers. Physical Review B, 2011. 83(20): p. 205429.

[13] Gupta, B., M. Notarianni, N. Mishra, M. Shafiei, F. Iacopi, and N. Motta, Evolution of epitaxial graphene layers on 3C SiC/Si (111) as a function of annealing temperature in UHV. Carbon, 2014. 68: p. 563-572.

[14] Brar, V.W., Y. Zhang, Y. Yayon, T. Ohta, J.L. McChesney, A. Bostwick, E. Rotenberg, K. Horn, and M.F. Crommie, Scanning tunneling spectroscopy of inhomogeneous electronic structure in monolayer and bilayer graphene on SiC. Applied Physics Letters, 2007. 91(12): p. 122102.

[15] Ridene, M., J.C. Girard, L. Travers, C. David, and A. Ouerghi, STM/STS investigation of edge structure in epitaxial graphene. Surface Science, 2012. 606(15-16): p. 1289-1292.

[16] Li, L. and I. Tsong, Atomic structures of 6H SiC (0001) and (0001) surfaces. Surface Science, 1996. 351(1): p. 141-148.

[17] Stolyarova, E., K.T. Rim, S. Ryu, J. Maultzsch, P. Kim, L.E. Brus, T.F. Heinz, M.S. Hybertsen, and G.W. Flynn, High-resolution scanning tunneling microscopy imaging of mesoscopic graphene sheets on an insulating surface. Proceedings of the National Academy of Sciences, 2007. 104(22): p. 9209.

[18] Van Bommel, A., J. Crombeen, and A. Van Tooren, LEED and Auger electron observations of the SiC (0001) surface. Surface Science, 1975. 48(2): p. 463-472.

[19] Starke, U., J. Schardt, J. Bernhardt, M. Franke, K. Reuter, H. Wedler, K. Heinz, J. Furthmüller, P. Käckell, and F. Bechstedt, Novel reconstruction mechanism for danglingbond minimization: Combined method surface structure determination of SiC (111)-(3×3). Physical review letters, 1998. 80(4): p. 758.

[20] Northrup, J.E. and J. Neugebauer, Theory of the adatom-induced reconstruction of the SiC(0001) $\sqrt{3} \times \sqrt{3}$ surface. Physical Review B, 1995. 52(24): p. R17001-R17004.

[21] Owman, F. and P. Mårtensson, STM study of the SiC (0001) $\sqrt{3} \times \sqrt{3}$ surface. Surface science, 1995. 330(1): p. L639-L645.

[22] Sabisch, M., P. Krüger, and J. Pollmann, Ab initio calculations of structural and electronic properties of 6H-SiC (0001) surfaces. Physical Review B, 1997. 55(16): p. 10561.

[23] Starke, U. and C. Riedl, Epitaxial graphene on SiC (0001) and SiC(000-1): from surface reconstructions to carbon electronics. Journal of Physics: Condensed Matter, 2009. 21(13): p. 134016.

[24] Lauffer, P., K. Emtsev, R. Graupner, T. Seyller, L. Ley, S. Reshanov, and H. Weber, Atomic and electronic structure of few-layer graphene on SiC (0001) studied with scanning tunneling microscopy and spectroscopy. Physical Review B, 2008. 77(15): p. 155426. 
[25] Riedl, C., C. Coletti, and U. Starke, Structural and electronic properties of epitaxial graphene on SiC (0 00 1): a review of growth, characterization, transfer doping and hydrogen intercalation. Journal of Physics D: Applied Physics, 2010. 43: p. 374009.

[26] Schardt, J., J. Bernhardt, U. Starke, and K. Heinz, Crystallography of the $(3 \times 3)$ surface reconstruction of 3 C-SiC (111), 4 H-SiC (0001), and 6 H-SiC (0001) surfaces retrieved by low-energy electron diffraction. Physical Review B, 2000. 62(15): p. 10335.

[27] Starke, U., J. Schardt, and M. Franke, Morphology, bond saturation and reconstruction of hexagonal SiC surfaces. Applied Physics A: Materials Science \& Processing, 1997. 65(6): p. 587-596.

[28] Ouerghi, A., A. Kahouli, D. Lucot, M. Portail, L. Travers, J. Gierak, J. Penuelas, P. Jegou, A. Shukla, and T. Chassagne, Epitaxial graphene on cubic SiC (111)/Si (111) substrate. Applied Physics Letters, 2010. 96: p. 191910-3.

[29] Dimitrakopoulos, C., Y.-M. Lin, A. Grill, D.B. Farmer, M. Freitag, Y. Sun, S.-J. Han, Z. Chen, K.A. Jenkins, and Y. Zhu, Wafer-scale epitaxial graphene growth on the Si-face of hexagonal SiC (0001) for high frequency transistors. Journal of Vacuum Science \& Technology B, 2010. 28(5): p. 985-992.

[30] Hass, J., J. Millán-Otoya, P. First, and E. Conrad, Interface structure of epitaxial graphene grown on 4H-SiC (0001). Physical Review B, 2008. 78(20): p. 205424.

[31] De Heer, W.A., C. Berger, X. Wu, P.N. First, E.H. Conrad, X. Li, T. Li, M. Sprinkle, J. Hass, and M.L. Sadowski, Epitaxial graphene. Solid State Communications, 2007. 143(1): p. 92-100.

[32] Hiebel, F., P. Mallet, F. Varchon, L. Magaud, and J. Veuillen, Graphene-substrate interaction on 6 H-SiC (000 1'): A scanning tunneling microscopy study. Physical Review B, 2008. 78(15): p. 153412.

[33] Hibino, H., H. Kageshima, and M. Nagase, Epitaxial few-layer graphene: towards single crystal growth. Journal of Physics D: Applied Physics, 2010. 43(37): p. 374005.

[34] Magaud, L., F. Hiebel, F. Varchon, P. Mallet, and J.-Y. Veuillen, Graphene on the Cterminated SiC (000 1) surface: An ab initio study. Physical Review B, 2009. 79(16): p. 161405.

[35] de Heer, W.A., C. Berger, M. Ruan, M. Sprinkle, X. Li, Y. Hu, B. Zhang, J. Hankinson, and E. Conrad, Large area and structured epitaxial graphene produced by confinement controlled sublimation of silicon carbide. Proceedings of the National Academy of Sciences, 2011. 108(41): p. 16900-16905.

[36] Aryal, H.R., K. Fujita, K. Banno, and T. Egawa, Epitaxial Graphene on Si (111) Substrate Grown by Annealing 3C-SiC/Carbonized Silicon. Japanese Journal of Applied Physics, 2012. 51(1S): p. 01AH05. 
[37] Ide, T., Y. Kawai, H. Handa, H. Fukidome, M. Kotsugi, T. Ohkochi, Y. Enta, T. Kinoshita, A. Yoshigoe, and Y. Teraoka, Epitaxy of Graphene on 3C-SiC (111) Thin Films on Microfabricated Si (111) Substrates. Japanese Journal of Applied Physics, 2012. 51(6S): p. 06FD02.

[38] Takahashi, R., H. Handa, S. Abe, K. Imaizumi, H. Fukidome, A. Yoshigoe, Y. Teraoka, and M. Suemitsu, Low-Energy-Electron-Diffraction and X-ray-Phototelectron-Spectroscopy Studies of Graphitization of 3C-SiC (111) Thin Film on Si (111) Substrate. Japanese Journal of Applied Physics, 2011. 50(7): p. 0103.

[39] Suemitsu, M. and H. Fukidome, Epitaxial graphene on silicon substrates. Journal of Physics D: Applied Physics, 2010. 43(37): p. 374012.

[40] Mårtensson, P., F. Owman, and L. Johansson, Morphology, Atomic and Electronic Structure of 6H-SiC (0001) Surfaces. physica status solidi (b), 1997. 202(1): p. 501-528.

[41] Riedl, C., U. Starke, J. Bernhardt, M. Franke, and K. Heinz, Structural properties of the graphene-SiC (0001) interface as a key for the preparation of homogeneous large-terrace graphene surfaces. Physical Review B, 2007. 76(24): p. 245406.

[42] Hannon, J. and R. Tromp, Pit formation during graphene synthesis on SiC (0001): In situ electron microscopy. Physical Review B, 2008. 77(24): p. 241404.

[43] Kaplan, R., Surface structure and composition of $\beta$-and 6H-SiC. Surface Science, 1989. 215(1): p. 111-134.

[44] Darakchieva, V., A. Boosalis, A. Zakharov, T. Hofmann, M. Schubert, T. Tiwald, T. Iakimov, R. Vasiliauskas, and R. Yakimova, Large-area microfocal spectroscopic ellipsometry mapping of thickness and electronic properties of epitaxial graphene on Si-and C-face of 3C-SiC (111). Applied Physics Letters, 2013. 102(21): p. 213116.

[45] Abe, S., H. Handa, R. Takahashi, K. Imaizumi, H. Fukidome, and M. Suemitsu, Surface Chemistry Involved in Epitaxy of Graphene on 3C-SiC (111)/Si (111). Nanoscale research letters, 2010. 5(12): p. 1888-1891.

[46] Suemitsu, M., Y. Miyamoto, H. Handa, and A. Konno, Graphene formation on a 3C-SiC (111) thin film grown on Si (110) substrate. e-Journal of Surface Science and Nanotechnology, 2009. 7(0): p. 311-313.

[47] Wang, L., S. Dimitrijev, J. Han, A. Iacopi, L. Hold, P. Tanner, and H.B. Harrison, Growth of 3C-SiC on 150-mm Si (100) substrates by alternating supply epitaxy at $1000^{\circ} \mathrm{C}$. Thin solid films, 2011. 519(19): p. 6443-6446.

[48] Gupta, B., M. Notarianni, N. Mishra, M. Shafiei, F. Iacopi, and N. Motta, Corrigendum to "Evolution of epitaxial graphene layers on 3C SiC/Si (111) as a function of annealing temperature in UHV" [Carbon 68 (2014) 563-572]. Carbon, 2015. 84(0): p. 280. 
[49] Giannozzi, P., S. Baroni, N. Bonini, M. Calandra, R. Car, C. Cavazzoni, D. Ceresoli, G.L. Chiarotti, M. Cococcioni, and I. Dabo, QUANTUM ESPRESSO: a modular and open-source software project for quantum simulations of materials. Journal of Physics: Condensed Matter, 2009. 21(39): p. 395502.

[50] Tersoff, J.H., D. R., Theory of the scanning tunneling microscope. Physical Review B 1985. 31: p. pp805-813 